\title{
Satisfação de Pacientes com Estomias Intestinais Quanto ao Uso de Equipamentos*
}

\author{
Patients with Intestinal Ostomy's Satisfaction in Using Equipments
}

\author{
Satisfacción de Pacientes con Estomas Intestinales Respecto al Uso de Medicamentos
}

\author{
Rosemary Vieira Souza Spenazato', Cláudia Regina de Souza Santos², \\ Ana Beatriz Pinto da Silva Morita ${ }^{3}$, Maria Angela Boccara de Paula ${ }^{3}$
}

\begin{abstract}
RESUMO
O estudo teve como objetivo avaliar o nível de satisfação dos pacientes com estomia intestinal relativo aos equipamentos adquiridos em um programa de assistência a estomizados de uma cidade do sul de Minas Gerais, por meio de abordagem quantitativa. Os dados foram coletados mediante entrevistas semiestruturadas com 32 pessoas. A média de idade foi de 61,6 anos, $68,75 \%$ dos pacientes eram casados, do sexo feminino (56,25\%), com estomas no quadrante inferior esquerdo (QIE) (43,75\%), 12\% devido à neoplasia, média de tempo de 6,2 anos, 75\% com colostomia, 93,75\% definitivos e 62,5\% apresentaram dermatite. Do total de participantes, 53,13\% usavam 2 peças; 84,38\%, peça opaca; e 96,87\%, peças drenáveis; média de 8 equipamentos ao mês; 96,87\% estavam satisfeitos com a quantidade. Os pacientes consideraram "Boas" as variáveis relativas à aderência durante o uso (59,38\%), à flexibilidade da base aderente (65,62\%) e à segurança no fechamento (59,38\%); foram encontrados valores iguais para "Boa" e "Ótima" (50\%) quanto à aderência durante a aplicação. Do total de participantes, o adesivo foi considerado confortável por 75\%, e 62,5\% dos pacientes não relataram odor; $56,25 \%$ relataram ausência de ruído durante o movimento; $75 \%$ consideraram fácil a higienização e a remoção (93,75\%); 75\% relataram não sentir dor na remoção; 65,63\% reportaram encontrar pouco resíduo após a retirada; 65,63\% permaneciam com o equipamento de 4 a 6 dias. Referente às orientações antes e após a cirurgia, $81,25 \%$ não as receberam antes e $62,5 \%$ não as receberam após a cirurgia enquanto hospitalizados; e todos (100\%) as receberam no Ambulatório de Estomaterapia. Os resultados, em sua maioria, foram de caráter positivo, presumindo-se que as pessoas com estomias intestinais possuem um nível de satisfação "Bom" quanto aos equipamentos fornecidos.
\end{abstract}

DESCRITORES: Equipamento. Estomia. Satisfação do paciente.

\begin{abstract}
The study was designed to evaluate the level of satisfaction of patients with intestinal stoma on the equipment purchased in a stomized assistance program of a city in the south of Minas Gerais, through a quantitative approach. Data were collected by semi-structured interviews with 32 people. The average age was 61.6 years, married (68.75\%), female (56.25\%), with stomas in the QIE (43.75\%), 12\% due to neoplasia, the average of time is 6.2 years, $75 \%$ with colostomy, $93.75 \%$ definitive and $62.5 \%$ showed dermatitis. Of the patients, $53.13 \%$ used two pieces, $84.38 \%$ opaque, $96.87 \%$ drainable, average of 8 devices per month and $96.87 \%$ were satisfied with the amount. Patients considered "Good" the variables related to adherence during use (59.38\%), flexibility of adherent base (65.62\%), security at closing (59.38\%) and values equal to "Good" and "Great" (50\%) in adherence during application. The adhesive was considered comfortable for $75 \%$ and $62.5 \%$, with no odor, 56.25\% related no noise during movement, $75 \%$ considered easy cleaning and removal $(93.75 \%)$, $75 \%$ no pain in removing, $65.63 \%$ a little bit of residue after removal and $65.63 \%$ remained with the equipment from 4 to 6 days. Referring to guidelines before and after surgery, $81.25 \%$ not received them before the surgery, and $62.5 \%$ not after, while hospitalized; and all (100\%) received them in Stomatherapy Clinic. Most of the results were of positive character, assuming that people with intestinal stoma have a level of satisfaction "Good" as the supplied equipment.
\end{abstract}

DESCRIPTORS: Equipment and supplies. Ostomy. Patient satisfaction.

\footnotetext{
*Artigo extraído de monografia de mesmo título referente à conclusão do Curso de Especialização de Enfermagem em Estomaterapia pela Universidade de Taubaté (UNITAU), para obtenção do título de Especialista em Estomaterapia, abril de 2015.

${ }^{1}$ Enfermeira Estomaterapeuta Consultora na Prática Assistencial de Enfermagem - Home Care - Pouso Alegre (MG), Brasil. Endereço para correspondência: Rua General Newton Marques de Azevedo, 135 - Fátima 1 - CEP: 37550-000 - Pouso Alegre (MG), Brasil - E-mail: rosemaryspenazato@hotmail.com ${ }^{2}$ Coordenadora do Ambulatório de Estomaterapia da Secretaria de Saúde de Pouso Alegre - Pouso Alegre (MG), Brasil.

${ }^{3}$ Coordenadora do Curso de Especialização em Enfermagem em Estomaterapia da Universidade de Taubaté (UNITAU) - Taubaté (SP), Brasil. Artigo recebido em: 29/02/2016 - Aceito para publicação em: 27/05/2016
} 


\section{RESUMEN}

El estudio tuvo como objetivo evaluar el nivel de satisfacción de los pacientes con estoma intestinal en em relación a los equipos adquiridos en un programa de asistencia para estomizados de una ciudad del sur de Minas Gerais, por medio de un abordaje cuantitativo. Los datos fueron recolectados por médio de entrevistas semiestructuradas con 32 personas. La edad media fue de 61,6 años, de pacientes casados (68,75\%), del sexo femenino (56,25\%), con estomas en el QIE (43,75\%), 12\% debido a la neoplasia, media de tiempo de 6,2 años, 75\% con colostomía, 93,75\% definitivos y $62,5 \%$ presentaron dermatitis. Del total de pacientes 53,13\%, usaban dos piezas, 84,38\% pieza opaca, 96,87\% piezas drenables, media de ocho equipos al mes y $96,87 \%$ estaban satisfechos con la cantidad. Los pacientes consideraron "Buenas" las variables relativas a la adherencia durante su uso (59,38\%), la flexibilidad de la base adherente (65,62\%), y la seguridad al cerrar (59,38\%) fueron encontrados valores iguales a "Buena" y "Óptima" (50\%) respecto a la adhesión durante la aplicación. El adhesivo fue considerado confortable por 75\% definitivos y 62,5\% com ausência de olor, 56,25\% relataron ausência de ruido durante el movimiento, 75\% considera fácil la higiene y remoción (93,75\%), 75\% relataron no sentir dolor en la remoción, 65,63\% reportaron encontrar poco residuo después de la retirada y 65,63\% permanecían con los equipos de 4 a 6 días. En referencia a las orientaciones antes y después de la cirugía, 81.25\% no las recibieron antes de la cirugía ni después (62,5\%), mientras estaban hospitalizados todos (100\%) la recibieron en el Ambulatorio de Estomaterapia. La mayoría de los resultados fueron de carácter positivo, presumiéndose que las personas con estomas intestinales tienen un nivel de satisfacción "Bueno" respecto a los equipos proporcionados.

DESCRIPTORES: Equipo. Estomía. Satisfacción del paciente.

\section{INTRODUÇÃO}

A pessoa com estomia intestinal enfrenta vários desafios, entre os quais se destaca a adaptação ao equipamento (bolsa coletora). Diante dessa situação, é importante que o enfermeiro, estomaterapeuta (ET) ou não, tenha conhecimento, para que possa atuar na escolha do tipo de equipamento ideal.

A colostomia se caracteriza por uma abertura realizada na parede cólica e exteriorizada através da parede abdominal anterior, com o intuito de desviar o efluente fecal para o meio exterior ${ }^{1}$.

As estomias podem fazer parte do tratamento de doenças malignas, benignas, inflamatórias, traumáticas e congênitas do trato gastrintestinal; para que haja uma adequada confecção, é necessário um planejamento pré-operatório e observação de detalhes técnicos, a fim de evitar complicações. É necessária uma estomia bem confeccionada que facilite o autocuidado, favorecendo, assim, a reabilitação ${ }^{2}$.

A tecnologia empregada nos equipamentos para estomias proporciona melhoria significativa da qualidade de vida dessas pessoas, minimizando, dessa forma, as alterações do estilo de vida, bem como do tipo de intervenção a que foram submetidas ${ }^{3}$.

Os equipamentos para estomias merecem atenção por serem o meio pelo qual as eliminações são coletadas e por causarem dependência por parte dos pacientes. Eles são encontrados fechados ou drenáveis, de uma ou duas peças (base adesiva e bolsa), transparentes ou opacos, recortáveis ou précortados, tanto para coleta de fezes como de urina. Mediante tal importância, eles são desenvolvidos para proporcionar segurança, facilitar o manuseio, permitir mobilização sem ruídos e, assim, tornar o convívio das pessoas com estomias mais harmonioso nos diversos âmbitos da vida, auxiliando também no retorno às atividades diárias ${ }^{4}$.

Para alguns autores ${ }^{3-5}$, a utilização adequada do equipamento deve ser indicada por enfermeiro ET ou enfermeiro capacitado para esse procedimento, por implicar na variedade e na escolha adequada para melhor fixação e adaptação, além de benefícios como conforto, segurança, praticidade, proteção da pele e permanência na região periestoma.

A visão do paciente sobre o uso da bolsa coletora implica em mudanças no aspecto cotidiano, constituindo um desafio para sua adaptação, marcado por momento de luto, por vezes patológico, devido às perdas sofridas. Além disso, essa nova situação exigirá, tanto da pessoa com estomia como dos familiares e amigos, sentimentos capazes de minimizar a realidade vivenciada e aceitação da nova situação ${ }^{5}$.

As dificuldades encontradas pelas pessoas com estomias para a realização das atividades de autocuidado estão relacionadas à insuficiência ou à ausência de orientações quanto ao manuseio no período pós-operatório, ocasionando talvez complicações na estomia e pele periestoma ${ }^{6}$.

$O$ interesse em saber sobre a satisfação com os equipamentos para estomias surgiu durante a realização de atendimento voluntário em um Ambulatório de Estomaterapia de um município do sul de Minas Gerais, em acompanhamento de pacientes com estomias intestinais nas consultas 
de enfermagem, pois alguns deles relatavam particularidades por alguns equipamentos.

Diante do exposto, o objetivo deste estudo foi avaliar o nível de satisfação dos pacientes com estomia intestinal (colostomia e ileostomia) em relação aos equipamentos adquiridos em um programa de assistência a estomizados de uma cidade do sul de Minas Gerais.

\section{MÉTODO}

Trata-se de um estudo descritivo e quantitativo realizado no Ambulatório de Estomaterapia da prefeitura de uma cidade do sul de Minas Gerais, o qual funciona como centro de referência às pessoas com estomias intestinais e urinárias.

A população da pesquisa foi composta por 97 pacientes com estomia intestinal (colostomia e ileostomia), cadastrados no Programa de Assistência a Pessoas com Estomias Intestinais e Urinárias do Ambulatório de Estomaterapia, sendo a amostra composta por 32 pessoas, maiores de 18 anos, com condições cognitivas e mentais para preencher o formulário, com estomia intestinal e que aceitaram participar da pesquisa assinando o Termo de Consentimento Livre e Esclarecido (TCLE). Foram excluídos da pesquisa aqueles que não residiam na cidade onde foi realizado o estudo, os que estavam acamados e os que tinham déficit cognitivo ${ }^{7}$.

A coleta de dados foi realizada no período de setembro a outubro de 2014, por meio de um instrumento elaborado pelas autoras com perguntas fechadas e abertas que constava de dados sociodemográficos, clínicos e relativos ao recebimento de orientações quanto à estomia e sugestões dos pacientes quanto aos equipamentos. Ela foi realizada após aprovação do Comitê de Ética e Pesquisa da Universidade de Taubaté (UNITAU), com CAAE no 792.196, de 12 de setembro de 2014.

O procedimento para a coleta dos dados foi realizado mediante uma reunião com pacientes com estomias intestinais que ocorria mensalmente no centro de referência às pessoas com estomias. Aqueles pacientes que por algum motivo não compareceram à reunião foram informados por contato telefônico e indagados sobre a participação no estudo em suas residências, com data e horário estipulados pelos participantes.

Os equipamentos fornecidos às pessoas com estomias são enviados pela Secretaria de Estado da Saúde de Belo Horizonte (SES-MG) a cada dois meses e entregues diretamente no centro de referência às pessoas com estomias. Essa entrega, conforme informações da enfermeira responsável, pode ser prejudicada por problemas relativos aos órgãos públicos, por fiscalização em portos, devido aos equipamentos serem importados, por empresa de transporte e por questões administrativas. Nesse caso, os pacientes são avisados sobre os problemas, e outros equipamentos, que não sejam os preferenciais, são oferecidos, com o intuito de não descontinuar seu uso e de evitar complicações com a pele.

Os resultados foram colocados em planilhas do programa Excel e quantificados em números absolutos e percentuais.

\section{RESULTADOS E DISCUSSÃO}

Dos 97 pacientes cadastrados desde o início do envio do projeto de pesquisa, 51 residiam no município onde foi realizado o estudo; desses, 32 participaram da pesquisa. A não participação foi decorrente de três deles possuírem urostomia, sete terem falecido, dois estarem acamados, dois estarem viajando, um estar internado, dois terem revertido, um ter mudado para outra cidade e um não ser encontrado em casa, apesar de dois retornos.

Os resultados apresentados nas Tabelas de 1 a 6 referem-se, respectivamente, aos seguintes dados sociodemográficos: distribuição das características clínicas dos pacientes; características dos equipamentos; dados sobre o grau de satisfação; dados sobre o recebimento de orientações; e sugestões quanto ao equipamento fornecido.

$\mathrm{Na}$ Tabela 1, observa-se o predomínio de mulheres (18/56,25\%), faixa etária entre 71 e 80 anos (12/37,5\%), média de idade de 61,6 anos e casados (22/68,75\%).

Quanto à média de idade, em um estudo realizado no Hospital de Ensino de Brasília, Distrito Federal, (2011) para verificar o conhecimento de 15 pessoas com estomia intestinal acerca dos cuidados e das orientações recebidos no pré e pós-operatório, houve o predomínio de $60 \%$ de mulheres, com média de idade de 48,33 anos ${ }^{8}$, menor do que a encontrada neste estudo.

Por meio de estudo retrospectivo ${ }^{9}$ realizado em 2013, após consulta em 308 prontuários de pacientes cadastrados em um programa de assistência a pessoas com estomias de uma cidade do sul de Minas Gerais, verificou-se que a média de idade (59 anos) foi semelhante à deste estudo, diferindo quanto ao sexo, no qual predominou o masculino (63\%). Em outros estudos ${ }^{10,11}$ o sexo masculino obteve 
a maior frequência (53\%), com média de idade de 56,3 anos e maior ocorrência do sexo feminino ${ }^{12}$.

Alguns estudos apontaram para pessoas casadas ${ }^{13-17}$, informação também reportada nesta pesquisa, não implicando na manutenção da intimidade do casal, assunto não abordado neste estudo.

Durante o estágio voluntário realizado em um serviço de atendimento às pessoas com estomas, percebeu-se um déficit em abranger a sexualidade durante as consultas de enfermagem. Por outro lado, ao frequentar encontros de pessoas com estomas realizados mensalmente, alguns pacientes mostraram-se desinibidos em falar sobre a intimidade do casal, relatando que achavam até engraçado o ruído do atrito do plástico do equipamento durante o ato sexual. Outros se mantiveram reservados, citando apenas que o estoma não os impedia de ter relações sexuais.

Tabela 1. Distribuição dos dados sociodemográficos de pessoas com estomia intestinal atendidas no Ambulatório de Estomaterapia de uma cidade do sul de Minas Gerais, 2014.

\begin{tabular}{|c|c|c|}
\hline Dados sociodemográficos & $\mathrm{n}$ & $\%$ \\
\hline \multicolumn{3}{|l|}{ Idade (em anos) } \\
\hline 31 a 40 & 1 & 3,13 \\
\hline 41 a 50 & 2 & 6,25 \\
\hline 51 a 60 & 8 & 25 \\
\hline 61 a 70 & 6 & 18,75 \\
\hline 71 a 80 & 12 & 37,5 \\
\hline 81 a 90 & 3 & 9,37 \\
\hline Total & 32 & 100 \\
\hline $\begin{array}{l}\text { Média de idade dos pacientes } \\
\text { (em anos) }\end{array}$ & \multicolumn{2}{|c|}{61,6} \\
\hline \multicolumn{3}{|l|}{ Sexo } \\
\hline Feminino & 18 & 56,25 \\
\hline Masculino & 14 & 43,75 \\
\hline Total & 32 & 100 \\
\hline \multicolumn{3}{|l|}{ Situação conjugal } \\
\hline Casado & 22 & 68,75 \\
\hline Solteiro & 2 & 6,25 \\
\hline Separado & 2 & 6,25 \\
\hline Viúvo & 6 & 18,75 \\
\hline Total & 32 & 100 \\
\hline
\end{tabular}

Fonte: formulário de pesquisa.
Do total de participantes, 25 (78,12\%) tiveram neoplasia e média de tempo com estomia de 6,2 anos, 24 (75\%) com colostomia, 14 (43,75\%) das estomias estavam localizadas no quadrante inferior esquerdo (QIE) e 20 (62,5\%) apresentaram complicações: 11 (34,4\%) eram dermatite, e 30 (93,75\%), definitivas (Tabela 2).

Tabela 2. Distribuição das características clínicas das pessoas com estomia intestinal atendidas no Ambulatório de Estomaterapia de uma cidade do sul de Minas Gerais, 2014.

\begin{tabular}{|c|c|c|}
\hline Dados clínicos & $\mathrm{n}$ & $\%$ \\
\hline \multicolumn{3}{|l|}{ Etiologia da estomia } \\
\hline Neoplasia & 25 & 78,12 \\
\hline Diverticulite & 4 & 12,5 \\
\hline Outros & 3 & 9,38 \\
\hline Total & 32 & 100 \\
\hline $\begin{array}{l}\text { Média de tempo com a } \\
\text { estomia (em anos) }\end{array}$ & \multicolumn{2}{|c|}{6,2} \\
\hline \multicolumn{3}{|l|}{ Tipo de estomia } \\
\hline Colostomia & 24 & 75 \\
\hline Ileostomia & 8 & 25 \\
\hline Total & 32 & 100 \\
\hline \multicolumn{3}{|l|}{ Localização da estomia } \\
\hline QID & 8 & 25 \\
\hline QSD & 3 & 9,37 \\
\hline QSE & 2 & 6,25 \\
\hline QIE & 14 & 43,75 \\
\hline Linha da cintura & 5 & 15,63 \\
\hline Total & 32 & 100 \\
\hline \multicolumn{3}{|l|}{ Presença de complicações* } \\
\hline Sem complicações & 12 & 37,5 \\
\hline Com complicações & 20 & 62,5 \\
\hline Dermatite* & 11 & 34,4 \\
\hline Hérnia paracolostômica* & 10 & 31,25 \\
\hline Retração* & 3 & 9,37 \\
\hline Granuloma* & 3 & 9,37 \\
\hline Prolapso* & 1 & 3,13 \\
\hline \multicolumn{3}{|l|}{ Permanência da estomia } \\
\hline Definitivo & 30 & 93,75 \\
\hline Temporário & 2 & 6,25 \\
\hline Total & 32 & 100 \\
\hline
\end{tabular}

Fonte: formulário de pesquisa.

*Admite-se mais de uma resposta; QID: quadrante inferior direito; QSD: quadrante superior direito; QSE: quadrante superior esquerdo; QIE: quadrante inferior esquerdo. 
Quanto ao tempo de permanência com a estomia, 14\% dos pacientes possuíam estomia há mais de 3 anos e 50\% possuíam colostomia definitiva ${ }^{11}, 38 \%$ com $\geq 38$ meses $^{17}, 58,3 \% \geq 16$ anos $^{15}$ e outro estudo ${ }^{20}$ diferindo com $73,7 \%$ com pessoas apresentando estomias há mais de 1 ano com caráter definitivo (75,2\%).

A dermatite periestoma é a complicação mais comum da pele circundante à estomia, é causada, geralmente, pelo contato com o efluente proveniente de ileostomia e colostomia direita, por problemas alérgicos, mecânicos e infecção; seu tratamento consiste na correção ou na eliminação do fator desencadeante ${ }^{18}$.

Ao estudar o cotidiano da pessoa com estomia diante das necessidades humanas básicas, a neoplasia colorretal (40\%) predominou entre outros diagnósticos ${ }^{10}$, situação também identificada em outra pesquisa, em que o índice foi de $51,4 \%{ }^{12}$. Quanto aos tumores de cólon e do reto, eles foram encontrados em mais de $60 \%$ dos entrevistados, em pessoas com estomia há mais de 1 ano (74\%), tipo colostomia $(88 \%)$ e, na maioria, casos permanentes ${ }^{13}$. Em pessoas com estomia definitiva (60\%), o câncer (73\%) foi predominante entre as causas de confecção da estomia intestinal, com tempo de permanência variando de 1 mês a 17 anos e com média de tempo de 4,33 anos ${ }^{8}$.

Um estudo realizado por meio de consulta a 178 prontuários de pacientes cadastrados em um programa de pessoas com estomias do Centro de Especialidades Médicas da Prefeitura Municipal de Campo Grande, Mato Grosso do Sul, mostrou que $85,4 \%$ foram colostomias e 46,6\% delas devido à neoplasia maligna, apresentando ainda complicações $(57,9 \%)$, com predominância da dermatite $(28,7 \%)^{19}$. Para alguns autores, a neoplasia também predominou entre as causas para realização da estomia intestinal ${ }^{19,15,20,13,11}$.

Um estudo ${ }^{9}$ mostrou que, entre as complicações encontradas nos pacientes, destacaram-se dermatite (16\%), retração $(10 \%)$, granuloma $(8 \%)$, com predominância daqueles em caráter temporário (51\%); outros estudos ${ }^{15-17}$ também apontaram que a dermatite foi predominante entre as complicações.

De acordo com a localização da estomia, os estudos ${ }^{6,16,17}$ apontam colostomia realizada no flanco esquerdo em 48, 31,65 e $45 \%$, respectivamente.

Conforme os resultados desta pesquisa, a presença de complicações chamou a atenção, devido ao fato de que pode interferir na vida diária desses pacientes. Apesar de não ter sido analisada a adequação do local do estoma, 5 (15,63\%) pessoas possuíam o dispositivo na linha da cintura. Supondo que a dobra de pele poderia estar presente, a dermatite seria a complicação mais frequente, como foi descrito no estudo. Além disso, a maior parte dos pacientes possuía estoma definitivo, 10 (31,25\%) deles com hérnia paracolostômica, sendo a cirurgia uma opção para correção, conforme desejo do paciente.

Quanto aos dados sobre os equipamentos utilizados pelos pacientes, dados apresentados na Tabela 3, 17 (53,13\%) faziam uso de equipamento de 2 peças, 27 (84,38\%), de peça opaca, 31 (96,87\%), de peças drenáveis e com mesmo valor para base adesiva plana, com média de 8 equipamentos por mês; os pacientes se declararam satisfeitos com essa quantia (31/96,87\%).

Em um estudo realizado no ano de $2007^{14}$ no município de Alfenas, Minas Gerais, cujo objetivo foi avaliar a qualidade de vida dos pacientes com estomias intestinais, dos 13 participantes, $61,5 \%$ relataram que a quantidade de bolsa era suficiente e $38,5 \%$ relataram que não era suficiente (valores não informados); referente aos equipamentos ${ }^{13}$, $6 \%$ tiveram problemas (dificuldades não especificadas).

Tabela 3. Distribuição dos equipamentos utilizados pelos pacientes com estomias intestinais atendidos em um Serviço de Estomaterapia de uma cidade do sul de Minas Gerais, 2014.

\begin{tabular}{|c|c|c|}
\hline Dados sobre equipamento & $\mathrm{n}$ & $\%$ \\
\hline \multicolumn{3}{|l|}{ Equipamento que faz uso } \\
\hline 1 peça & 15 & 46,87 \\
\hline 2 peças & 17 & 53,13 \\
\hline Total & 32 & 100 \\
\hline Opaca & 27 & 84,38 \\
\hline Transparente & 5 & 15,62 \\
\hline Total & 32 & 100 \\
\hline Drenável & 31 & 96,87 \\
\hline Fechada & 1 & 3,13 \\
\hline Total & 32 & 100 \\
\hline Base adesiva plana & 31 & 96,87 \\
\hline Base adesiva convexa & 1 & 3,13 \\
\hline Total & 32 & 100 \\
\hline $\begin{array}{l}\text { Média de equipamentos } \\
\text { fornecidos por mês (bolsa) }\end{array}$ & & B \\
\hline \multicolumn{3}{|c|}{ Satisfação com quantidade fornecida } \\
\hline $\operatorname{sim}$ & 31 & 96,87 \\
\hline Não & 1 & 3,13 \\
\hline Total & 32 & 100 \\
\hline
\end{tabular}


Ao caracterizar a clientela residente em Ponte Nova, Minas Gerais, amostra de 12 pessoas com estomias, o tipo de equipamento predominante entre os pacientes era de uma peça $(91,65 \%)$, peça drenável $(66,6 \%)$ e peça recortável $(91,6 \%)^{15}$. Talvez a maior quantidade de equipamentos de uma peça possa ser explicada devido ao predomínio da estomia em cólon esquerdo, em que a consistência das fezes pode variar entre pastosa e formada, além de o número de trocas ser de 1 a 2 vezes por semana. Além disso, todos os participantes mencionaram o uso adequado dos equipamentos, porém nem todas as expectativas eram atendidas (33,3\%).

Os resultados foram diferentes: a maior ocorrência se deu para equipamentos de 2 peças (base adesiva e bolsa - 74\%) e de cor opaca (85\%); os demais dados assemelharam-se ao estudo anterior, com predomínio de equipamentos recortáveis $(83,5 \%)$, drenáveis (98\%) e com base adesiva plana $(83,5 \%)^{17}$.

É importante que as pessoas que fazem uso de equipamentos para estomias tenham a oportunidade de escolher aquele que traz mais conforto e garanta sua segurança, independentemente da quantidade oferecida. Foi registrado um caso isolado de um paciente com ileostomia que preferia usar equipamento de uma peça com trocas diárias, apesar de ser orientado quanto ao tipo mais adequado, conforme relatou a enfermeira do serviço onde foi realizada esta pesquisa.

Por outro lado, apesar de a maioria das pessoas possuir colostomia, na qual as fezes se apresentam variando de pastosas a formadas, subentende-se que os equipamentos do tipo uma peça poderiam ser usados por grande parte dos pacientes. No entanto, observando as consultas de retorno dos pacientes, realizadas pela enfermeira ET, após o período de um a dois meses, ao questionar qual equipamento o paciente preferiu, obtinha como resposta "aquele de duas peças (placa adesiva e bolsa)", devido à praticidade para limpeza e trocas. Os equipamentos para estomia são imprescindíveis para a manutenção da qualidade de vida dessas pessoas. A convivência do enfermeiro com os pacientes leva a uma relação de confiança, proporcionando informações que se revertem em procedimentos que favorecem a adequação dos equipamentos coletores.

As variáveis das Tabelas 4 e 5 foram divididas para facilitar a visualização e discussão.

A Tabela 4 mostrou que os pacientes consideraram "Boas" as variáveis relativas à aderência durante o uso (19/59,38\%), à flexibilidade da base aderente $(21 / 65,62 \%)$ e à segurança no fechamento (19/59,38\%); valores iguais para "Bom" e
"Ótimo" (16/50\%) foram relativos à satisfação dos pacientes quanto à aderência durante a aplicação. $\mathrm{O}$ adesivo foi considerado confortável por 24 (75\%) pacientes, e 20 (62,5\%) relataram que não passava nenhum odor.

Em uma pesquisa realizada com 15 pacientes, os resultados mostraram que o equipamento coletor preferido por todos os entrevistados foi aquele que eles consideravam ser

Tabela 4. Dados sobre o grau de satisfação de pacientes com estomias intestinais atendidos em um Serviço de estomaterapia quanto à satisfação dos equipamentos com relação à aderência, à flexibilidade, à segurança, ao conforto e ao odor de uma cidade do sul de Minas Gerais, 2014.

$\begin{array}{lll}\text { Grau de satisfação } & \text { n } & \% \\ \text { quanto ao equipamento } & & \end{array}$

Aderência na aplicação

$\begin{array}{lll}\text { Bom } & 16 & 50 \\ \text { Ótimo } & 16 & 50 \\ \quad \text { Total } & 32 & 100\end{array}$

Aderência durante o uso

$\begin{array}{lll}\text { Bom } & 19 & 59,38 \\ \text { Ótimo } & 10 & 31,25 \\ \quad \text { Ruim } & 32 & 9,37 \\ \quad \text { Total } & 32 & 100\end{array}$

Flexibilidade da base aderente

\begin{tabular}{|c|c|c|}
\hline Bom & 21 & 65,62 \\
\hline Ótimo & 10 & 31,25 \\
\hline Ruim & 1 & 3,13 \\
\hline Total & 32 & 100 \\
\hline \multicolumn{3}{|c|}{ Segurança no fechamento } \\
\hline Bom & 19 & 59,38 \\
\hline Ótimo & 10 & 31,25 \\
\hline Ruim & 3 & 9,37 \\
\hline Total & 32 & 100 \\
\hline \multicolumn{3}{|c|}{ Conforto do adesivo } \\
\hline Confortável & 24 & 75 \\
\hline Razoável & 7 & 21,87 \\
\hline Desconfortável & 1 & 3,13 \\
\hline Total & 32 & 100 \\
\hline \multicolumn{3}{|l|}{ Passagem do odor } \\
\hline Nenhum & 20 & 62,5 \\
\hline Pouco & 10 & 31,25 \\
\hline Muito & 2 & 6,25 \\
\hline Total & 32 & 100 \\
\hline
\end{tabular}

Fonte: formulário de pesquisa. 
mais confortável, mais higiênico, menos alérgeno, de maior durabilidade, de maior aderência, que prevenisse odor e que fosse drenáve ${ }^{18}$.

Em vivência voluntária no Serviço de Estomaterapia foram observadas orientações pertinentes às variáveis

Tabela 5. Dados sobre o grau de satisfação de pacientes com estomias intestinais cadastrados em um Serviço de estomaterapia quanto à satisfação dos equipamentos relativos aos ruídos, à higienização, ao nível de dor na remoção, aos resíduos e ao tempo com a bolsa coletora de uma cidade do sul de Minas Gerais, 2014.

\begin{tabular}{|c|c|c|}
\hline $\begin{array}{l}\text { Grau de satisfação } \\
\text { quanto ao equipamento }\end{array}$ & $\mathrm{n}$ & $\%$ \\
\hline \multicolumn{3}{|c|}{ Ruído do plástico durante movimento } \\
\hline Nenhum & 18 & 56,25 \\
\hline Pouco & 14 & 43,75 \\
\hline Total & 32 & 100 \\
\hline \multicolumn{3}{|l|}{ Higienização } \\
\hline Fácil & 24 & 75 \\
\hline Razoável & 6 & 18,75 \\
\hline Difícil & 2 & 6,25 \\
\hline Total & 32 & 100 \\
\hline \multicolumn{3}{|l|}{ Remoção } \\
\hline Fácil & 30 & 93,75 \\
\hline Razoável & 2 & 6,25 \\
\hline Total & 32 & 100 \\
\hline \multicolumn{3}{|l|}{ Nível de dor na remoção } \\
\hline Ausente & 24 & 75 \\
\hline Tolerável & 8 & 25 \\
\hline Total & 32 & 100 \\
\hline \multicolumn{3}{|c|}{ Presença de resíduo do adesivo na pele } \\
\hline Nenhum & 8 & 25 \\
\hline Muito & 3 & 9,37 \\
\hline Pouco & 21 & 65,63 \\
\hline Total & 32 & 100 \\
\hline \multicolumn{3}{|l|}{ Tempo com equipamento } \\
\hline 1 a 3 dias & 9 & 28,12 \\
\hline 4 a 6 dias & 21 & 65,63 \\
\hline 7 dias & 2 & 6,25 \\
\hline Total & 32 & 100 \\
\hline
\end{tabular}

Fonte: formulário de pesquisa. da Tabela 4, as quais foram discutidas com a enfermeira do serviço em estomias. Em relação à permanência do equipamento, a profissional orientava sobre a importância da hidratação oral, além da ausência de produtos oleosos no local de aderência da base adesiva, da permanência em repouso por, no mínimo, 30 minutos após a colocação do equipamento, além de recortar o equipamento na medida correta, ou seja, com espaço de aproximadamente 2 a $3 \mathrm{~mm}$ entre o estoma e a base adesiva.

O item "flexibilidade" faz parte das indicações do profissional, no qual devem ser prescritas observando proximidade com proeminências ósseas, como crista ilíaca e rebordo costal. Ao passar para a segurança no fechamento, é importante proceder à demonstração do equipamento de forma detalhada aos familiares e pacientes, quantas vezes for necessário, durante a consulta de enfermagem. $\mathrm{O}$ conforto do adesivo é um item que será verificado, com o paciente e o equipamento de preferência, ao longo dos retornos, assim como será feita a avaliação da passagem de odor.

No que tange ao cuidado da pessoa com estomia, o enfermeiro se depara com uma gama de equipamentos e adjuvantes disponíveis no mercado para o atendimento. A indicação e a seleção do material adequado ao tipo de estoma e às características da pessoa e do efluente constituem etapa fundamental para o processo de reabilitação ${ }^{21 .}$

Os itens analisados quanto à aderência, ao conforto do adesivo e à passagem de odor tiveram considerações desfavoráveis neste estudo, podendo estar relacionados com a presença de complicações como a dermatite e a hérnia paraestomal. Na presença da hérnia paraestomal, devido à conformação côncava do local para aderência da base adesiva, pode haver dificuldade de aderência, causando vazamentos e passagens de odor, fatores também observados na dermatite, acrescida da ardência na pele.

Quanto ao ruído do plástico durante o movimento, 18 $(56,25 \%)$ pacientes relataram que não tinha nenhum ruído, 24 (75\%) consideraram fácil a higienização, 30 (93,75\%) acharam de fácil remoção, 24 (75\%) relataram ausência de dor na remoção, $21(65,63 \%)$ reportaram pouco resíduo após a retirada, e $21(65,63 \%)$ permaneciam com o equipamento de 4 a 6 dias (Tabela 5).

Quanto à higienização, 23\% dos pacientes encontraram dificuldade para a higienização da bolsa ${ }^{13}$, sendo também verificada a colocação inadequada da base adesiva $(50,6 \%)^{19}$, $66,7 \%$ dos pacientes tinham habilidade total (troca e higiene $)^{15}$ e $53,8 \%$ não apresentavam dificuldade ${ }^{14}$. 
Referente ao cuidado de enfermagem aos usuários com estomias, uma paciente mostrou-se insatisfeita, pois fazia um ano e cinco meses que fazia uso da bolsa e não saía mais de casa, afirmando ter vergonha e constrangimento na presença de barulho do equipamento e preocupação com a percepção de outras pessoas quanto a isso ${ }^{22}$. Outro estudo ${ }^{23}$ também abordou esse item em sua pesquisa, obtendo em uma das respostas que "às vezes quando eu vou para lugares com muitas pessoas, fico preocupada, porque a bolsa faz aquela zoadinha, ai vêm os gases, ai eu fico com receio de estourar, e me afasto". Alguns pacientes entrevistados no presente trabalho também referiram vergonha em ambiente público e que a colostomia começa a fazer barulho, como é possível perceber na fala, em tom de brincadeira: "isso acontece porque não tem mais freio".

Quanto à remoção, durante as entrevistas, foi praticamente unânime a facilidade em retirar, a não ser quando retornava ao médico e era pedido para retirar a bolsa, o que causava desconforto, pois não era oferecido nenhum tipo de lenço úmido ou gaze molhada. Após essa queixa, o paciente foi orientado a levar uma bolsa contendo frasco com sabonete diluído e outro com água, para diminuir a dor e o desconforto ao retirar a base adesiva, além de levar um equipamento para realizar a troca.

A presença de resíduos foi apontada por mais da metade dos pacientes. Isso talvez ocorra devido à boa aderência da base adesiva, dependendo também da época do ano e do clima, variando com baixas e altas temperaturas. Essa variável merece abordagem mais ampla, sendo um fator limitante para discussão neste estudo.

O tempo de equipamento pode variar com a presença de orientações e a qualidade delas. Foi observado durante a vivência voluntária que os próprios pacientes, durante os retornos, relatavam a quantidade de dias em que o equipamento permanecia e quais eram as condições em que desprendiam da pele. Ao contrário de outro estudo ${ }^{8}$ no qual nenhum entrevistado soube afirmar com precisão o tempo de duração do equipamento. Diante dos relatos, a enfermeira reorientava os pacientes, procurando encontrar em quais condições eles poderiam melhorar a colocação das bolsas.

A convivência com a bolsa de colostomia gera sentimentos conflituosos, preocupações e dificuldade para lidar com essa nova situação ${ }^{23}$. Talvez seja por isso que alguns pacientes prefiram trocar seus equipamentos mais vezes na semana, aumentando, com isso, a quantidade recebida ao mês.
A escolha do equipamento adequado é o elemento essencial para o sucesso no processo de reabilitação e reintegração social da pessoa com estomia,já que o dispositivo é um recurso indispensável ${ }^{21}$. Diante disso, o enfermeiro, ET ou não, precisa conhecer os produtos disponíveis no mercado, suas características e peculiaridades, bem como os recursos que a comunidade oferece para a continuidade da assistência.

A Tabela 6 mostra que $26(81,25 \%)$ pacientes não receberam orientações antes da cirurgia, e 20 (62,5\%) nem após a cirurgia; apenas no Ambulatório de Estomaterapia todos receberam orientações quanto ao manuseio e aos equipamentos (32/100\%).

Um estudo mostrou que $53,3 \%$ dos pacientes receberam orientações no pré-operatório e 26,6\% relataram não ter recebido nenhum tipo de orientação (não foram especificadas as orientações $)^{8}$. O processo educativo enfermeiro-paciente deve ter como principal objetivo o fornecimento de informações necessárias para o autocuidado da pessoa com estomia, a fim de reduzir medos, prevenir complicações pósoperatórias, alcançar sua reabilitação e seu bem-estar geral, e melhorar sua qualidade de vida. A ausência de orientações também foi descrita em uma pesquisa ${ }^{17}$, em que $69,4 \%$ dos pacientes não foram informados antes da cirurgia quanto aos tipos de equipamentos existentes, à higienização, à presença de grupo de pessoas com estomias, à alimentação,

Tabela 6. Dados sobre o recebimento de orientações quanto aos equipamentos, ao manuseio e à cirurgia de pacientes com estomas intestinais cadastrados em um Serviço de estomaterapia de uma cidade do sul de Minas Gerais, 2014.

\begin{tabular}{lcc}
\hline Recebimento de orientações & $\mathbf{n}$ & $\%$ \\
\hline Antes da cirurgia & & \\
\hline Sim & 26 & 18,75 \\
\hline Não & 31,25 \\
\hline Total & 100 \\
\hline Após a cirurgia & 12 & 37,5 \\
\hline Sim & 20 & 62,5 \\
Não & 32 & 100 \\
\hline Total & 32 & 100 \\
\hline Orientação ambulatorial & 32 & 100 \\
\hline Sim &
\end{tabular}


ao vestuário e ao relacionamento, ao cuidado com a pele e à troca de bolsa.

A consulta de enfermagem pré-operatória minimiza a ansiedade e o temor, esclarece dúvidas, pois frequentemente os pacientes não têm ideia de como é um estoma, do equipamento coletor (bolsa de colostomia) e de como conviverão com o estoma ${ }^{24}$.

Quanto aos equipamentos fornecidos, algumas sugestões foram relatadas:

- "Melhorar a aderência da base" (2/6,25\%);

- "Melhorar o encaixe da bolsa na placa" (1/3,13\%);

- "Aumentar o tamanho do adesivo microporoso"(2/6,25\%);

- "Aumentar tanto a quantidade de bolsa como de barreira em pasta fornecida" (3/9,37\%);

- "Ausência de filtro de carvão" (1/3,13\%);

- "Aumentar a quantidade apenas de bolsas, para não ter que lavar constantemente" (2/6,25\%).

Os dados acima referentes a "aumentar a quantidade de equipamentos fornecidos" contrariam as respostas obtidas na Tabela 3, na qual é possivel verificar que que 31 (96,87\%) pacientes estavam satisfeitos com a quantidade fornecida, bem como com a "ausência de filtro de carvão”. Esse é um dado que poderá ser investigado posteriormente, a fim de esclarecer a possível duplicidade de entendimento.

As sugestões referentes a "melhorar a aderência da base", "melhorar o encaixe da bolsa na placa" e "aumentar o tamanho do adesivo microporoso" foram repassadas para a enfermeira do Ambulatório de Estomaterapia para que fossem encaminhadas aos órgãos públicos responsáveis pelo fornecimento dos equipamentos. Além dessa ação, essas sugestões seriam discutidas em reuniões mensais, realizadas com os pacientes com estomias, reorientando o manuseio dos equipamentos.

\section{CONCLUSÃO}

Entre os resultados apresentados nesta pesquisa, verifica-se que: $68,75 \%$ dos pacientes eram casados, $56,25 \%$ eram do sexo feminino, com causa para a confecção da estomia, na maior parte dos casos $(78,12 \%)$, a ocorrência de neoplasia e média de idade de 61,6 anos. A maioria (75\%) com colostomia, 93,75\%, eram definitivos, e $62,5 \%$ apresentaram complicações, dentre elas, 34,4\% como dermatite. Referente aos equipamentos, 53,13\% faziam uso de equipamento de 2 peças, 96,87\%, drenáveis e com mesmo valor para base adesiva plana, com média de 8 equipamentos por mês, e 96,87\% estavam satisfeitos com essa quantidade.

Quanto ao nível de satisfação, os pacientes consideraram “Bons" os itens relativos à aderência durante o uso (59,38\%), à flexibilidade da base aderente $(65,62 \%)$, à segurança no fechamento $(59,38 \%)$, sendo encontrados valores iguais para "Boa" e "Ótima" (50\%) quanto à aderência durante a aplicação. Do total de participantes, o adesivo foi considerado o adesivo foi considerado confortável por $75 \%$, e $62,5 \%$ dos pacientes não relataram odor; $56,25 \%$ relataram ausência de ruído do plástico durante o movimento; $75 \%$ consideraram fácil a higienização; 93,75\% não encontraram dificuldade com a remoção; 75\% relataram ausência de dor na remoção; 65,63\% reportaram pouco resíduo após a retirada; e 65,63\% permaneciam com o equipamento de 4 a 6 dias.

Referente às orientações antes e após a cirurgia, 81,25\% dos pacientes não as receberam antes e 62,5\% não as receberam após a cirurgia enquanto hospitalizados, e todos (100\%) vieram a recebê-las no Ambulatório de Estomaterapia. Em relação às sugestões quanto aos equipamentos, algumas foram relatadas, como "melhorar a aderência da base", "melhorar o encaixe da bolsa na placa”, "aumentar o tamanho do adesivo microporoso", "aumentar tanto a quantidade de bolsa como de barreira em pasta fornecida", "ausência de filtro de carvão" e "aumentar a quantidade apenas de bolsas, para não ter que lavar constantemente".

A maioria dos resultados foi de caráter positivo, presumindo-se que as pessoas com estomias intestinais apresentam um nível de satisfação "Bom" quanto aos equipamentos fornecidos. O enfermeiro, ET ou não, tem uma função essencial no cuidado do paciente com estomia intestinal; por isso, deve orientá-lo sobre o uso da bolsa, tirando suas dúvidas e dando explicações sobre o cuidado com o equipamento, a higienização, o tempo de troca, assim como sobre possíveis complicações, incentivando-o sempre quanto à importância do autocuidado.

Percebe-se que este estudo forneceu informações importantes para melhorar o atendimento às pessoas com estomias intestinais. Outras pesquisas devem ser realizadas com a finalidade de fornecer dados para construção de diretrizes relativas ao programa de pacientes com estomias. 


\section{REFERÊNCIAS}

1. Paula PR, Speranzini MB. Colostomias e ileostomias. In: Boccara de Paula MA, Paula PR, Cesaretti IUR (orgs.). Estomaterapia em foco e o cuidado especializado. São Caetano do Sul: Yendis; 2014. p.18-32.

2. Paula PR. Situações clínicas que levam a confecção de estomas intestinais. In: Cesaretti IUR, Boccara de Paula MA, Paula PR (orgs.) Estomaterapia: temas básicos em estomas. Taubaté: Cabral; 2006. p.51-66.

3. Cascais AFMV, Martini JG, Almeida PJS. O impacto da ostomia no processo de viver humano. Rev Tex Cont Enf. 2007;16(1):163-7.

4. Marcondes MG. Características dos dispositivos para uso de estomas. In: Cesaretti IUR, Boccara de Paula MA, Paula PR (orgs.) Estomaterapia: temas básicos em estomas. Taubaté: Cabral; 2006. p.125-37.

5. Barbutti RCS, Silva MCP, Abreu MAL. Ostomia, uma difícil adaptação. Rev SBPH. 2008;11(2):27-39.

6. Yamada BFA, Cesaretti IUR, Marcondes MGSG, Morais JF, Prado AAB. Ocorrência de complicações no estoma e pele periestoma: estudo retrospectivo. ESTIMA. 2003;1(3):16-24.

7. Instituto Brasileiro de Geografia e Estatística (IBGE). Cidades; 2014. [Internet] [cited 2014 Oct 20]. Available from: http:// cidades.ibge.gov.br/xtras/perfil.php?Lang=\&comum=31525 0\&search=minas-gerais\%7Cpouso-alegre

8. Kamada I, Faustino AM, Silva AL, Vieira ABD, Borges CT. Conhecimento acerca de estomia intestinal por pacientes acompanhados em um serviço ambulatorial de enfermagem em estomaterapia: estudo qualitativo. ESTIMA. 2011;9(4):21-7.

9. Spenazato RVS, Santos CRS, Boccara de Paula MA, Matias JL, Santos JL, Morita ABS. Estomas complicados em pacientes de um ambulatório de estomaterapia de um município do sul de Minas Gerais: um estudo documental. In: CONGRESSO BRASILEIRO DE ESTOMATERAPIA, 10. Anais... Pouso Alegre: Sobest; 2013: p.45.

10. Galdino YLS, Castro ME, Pereira MLD, Lima SSO, Silva FAA, Guedes MVC. O cotidiano da pessoa estomizada frente às necessidades humanas básicas alteradas. ESTIMA. 2012;10(3):22-30

11. Meirelles CA, Ferraz CA. Avaliação da qualidade do processo de demarcação do estoma intestinal e das intercorrências tardias em pacientes ostomizados. Rev Latino-Am Enfermagem. 2001;9(5):32-8.

12. Mantovani MF, Muniz MR, Simões MC, Boschco MD, Oliveira GD. O perfil dos usuários cadastrados na associação paranaense de ostomizados. Cog Enf. 2007;12(1):76-81.
13. Bechara RN, Bechara MS, Bechara CS, Queiroz HC, Oliveira RB, Mota RS, et al. Abordagem multidisciplinar do ostomizado. Rev Bras Coloproct. 2005;25(2):146-9.

14. Esteves AMSD. Avaliação da qualidade de vida de pacientes portadores de estomas intestinais [dissertação]. Alfenas: Universidade Federal de Alfenas; 2009.

15. Fernandes RM, Miguir ELB, Donoso TV. Perfil da clientela estomizada residente no município de Ponte Nova, Minas Gerais. Rev Bras Col. 2010;30(4):385-92.

16. Pi Chilida MS, Santos AH, Calvo AMB, Bello BEC, Alves DA, Guerino MI. Complicações mais frequentes em pacientes atendidos em um pólo de atendimento ao paciente com estoma no interior de São Paulo. ESTIMA. 2007;5(4):31-6.

17. Lima GA, Sousa VF, Santos CRS. Caracterização de estomizados de um centrode referência de estomas intestinais e urinários de uma cidade no sul de minas gerais. In: CONGRESSO BRASILEIRO DE ESTOMATERAPIA, 10. Anais... Pouso Alegre: Sobest; 2012. p.74.

18. Scianni RC, Cesaretti IUR e Boccara de Paula MA. Estomas complicados: como cuidar. In: Paula PR, Paula MAB e Cesaretti IUR. Estomaterapia: Temas Básicos em Estomas. Taubaté: Cabral; 2006. p.137-58.

19. Santos CHM, Bezerra MM, Bezerra FMM, Paraguassu BR. Perfil do Paciente Ostomizado e Complicações Relacionadas ao Estoma. Rev Bras Coloproct. 2007;27(1):16-9.

20. Violin MR, Mathias TAF, Uchimura TT. Perfil de clientes colostomizados inscritos em programa de atenção aos estomizados. Rev Elet Enf. 2008;10(4):924-32.

21. Lage EMI, Boccara de Paula MA, Cesaretti IUR. Equipamentos coletores e adjuvantes usados no cuidado das estomias. In: Boccara de Paula MA, Paula PR, Cesaretti IUR (orgs.). Estomaterapia em foco e o cuidado especializado. São Caetano do Sul: Yendis; 2014. p.151-67.

22. Carvalho VMI, Cardoso JRS. Cuidados com dermatites periestomais. In: Malagutti W, Kakihara CT (orgs.). Curativos, estomia e dermatologia: Uma abordagem multiprofissional. São Paulo: Martinari; 2011. p.487-94.

23. Batista MRFF, Rocha FCV, Silva DMG, Silva Junior FJG. Autoimagem de clientes com colostomia em relação à bolsa coletora. Rev Bras Enf. 2011;64(6):1043-7.

24. Schwartz MP, Sá SPC, Santos FS, Santos MLSC, Valente GSC. O cuidado ao paciente no pré-operatório de estoma intestinal provisório: revisão integrativa de literatura. ESTMA. 2012;10(3):31-5. 